\title{
Sonification and haptic feedback in addition to visual feedback enhances complex motor task learning
}

\author{
Roland Sigrist • Georg Rauter • \\ Laura Marchal-Crespo - Robert Riener $\cdot$ Peter Wolf
}

Received: 30 October 2013 / Accepted: 27 November 2014 / Published online: 16 December 2014

(C) Springer-Verlag Berlin Heidelberg 2014

\begin{abstract}
Concurrent augmented feedback has been shown to be less effective for learning simple motor tasks than for complex tasks. However, as mostly artificial tasks have been investigated, transfer of results to tasks in sports and rehabilitation remains unknown. Therefore, in this study, the effect of different concurrent feedback was evaluated in trunk-arm rowing. It was then investigated whether multimodal audiovisual and visuohaptic feedback are more effective for learning than visual feedback only. Naïve subjects $(N=24)$ trained in three groups on a highly realistic virtual reality-based rowing simulator. In the visual feedback group, the subject's oar was superimposed to the target oar, which continuously became more transparent when the deviation between the oars decreased. Moreover, a trace of the subject's trajectory emerged if deviations exceeded a threshold. The audiovisual feedback group trained with oar movement sonification in addition to visual feedback to facilitate learning of the velocity profile. In the visuohaptic group, the oar movement was inhibited by path deviationdependent braking forces to enhance learning of spatial aspects. All groups significantly decreased the spatial error (tendency in visual group) and velocity error from baseline to the retention tests. Audiovisual feedback fostered learning of the velocity profile significantly more than visuohaptic feedback. The study revealed that well-designed concurrent feedback fosters complex task learning, especially if
\end{abstract}

R. Sigrist $(\bowtie) \cdot$ G. Rauter $\cdot$ L. Marchal-Crespo $\cdot$ R. Riener . P. Wolf

Sensory-Motor Systems (SMS) Lab, Institute of Robotics and Intelligent Systems (IRIS), ETH Zurich, Sonneggstrasse 3 (ML G 57), 8092 Zurich, Switzerland

e-mail: roland.sigrist@ hest.ethz.ch

R. Sigrist · G. Rauter $\cdot$ L. Marchal-Crespo $\cdot$ R. Riener $\cdot$ P. Wolf Medical Faculty, University of Zurich, Zurich, Switzerland the advantages of different modalities are exploited. Further studies should analyze the impact of within-feedback design parameters and the transferability of the results to other tasks in sports and rehabilitation.

Keywords Augmented feedback - Movement sonification · Haptic guidance - Multimodal feedback · Robot-assisted learning $\cdot$ Rowing simulator

\section{Introduction}

Finding effective ways to accelerate learning of movements is of interest in different fields such as surgery, motor rehabilitation, and sports. A widely discussed strategy for enhancing motor learning is the provision of augmented (extrinsic) feedback (Sigrist et al. 2013a; Wulf and Shea 2002), i.e., feedback about the results or performance that is provided by an external source such as a trainer, a therapist, or a display (Schmidt and Wrisberg 2008). Kinematic and kinetic variables of the human movement are fed back in different modalities by various displays, e.g., by visual displays, auditory displays (headphones, speakers), or haptic displays (robots) (Sigrist et al. 2013a). Most related studies have used visual feedback to investigate the effects of different feedback schedules, e.g., when and how often a feedback should be provided. Thereby, unimodal visual feedback was dominantly applied (Sigrist et al. 2013a). The latest sports simulators such as the rowing simulator used in this study (Rauter et al. 2010, 2013; von Zitzewitz et al. 2008) allow for the investigation of not only unimodal feedback, but also multimodal feedback of complex tasks in a controllable environment (Holden 2005; Sigrist et al. 2013a). The first aim of this study on rowing was to evaluate feedback designs that were developed based 
on the current knowledge of motor learning research and that exploited advantages of different sensory modalities. The second aim was to test the potential benefits of multimodal feedback in a task related to sports and rehabilitation deduced from basic research on perception and action (Alais and Burr 2004; Burke et al. 2006; Carson and Kelso 2004; Ernst and Banks 2002; Seitz and Dinse 2007; Shams and Seitz 2008; van Beers et al. 1999; Wickens 2002). It was tested whether the addition of auditory or haptic feedback to visual feedback would facilitate or hamper motor learning as compared to visual feedback alone. Intersensory facilitation, i.e., the more precise and faster perception of multimodal than unimodal stimuli (Carson and Kelso 2004), and multimodal integration, i.e., the optimal weighting of sensory information by the nervous system (Alais and Burr 2004; Ernst and Banks 2002; van Beers et al. 1999), may benefit the learning process. In contrast, additional feedback modalities may hamper learning due to cognitive overload or distraction (Sigrist et al. 2013a).

For simple tasks, frequent terminal augmented feedback (provided after task execution) or concurrent augmented feedback (provided during task execution) was found to increase performance during training. However, performance dropped dramatically when the feedback was withdrawn (Schmidt and Wulf 1997; van der Linden et al. 1993; Winstein et al. 1996). This phenomenon was described as the guidance hypothesis predicting the dependency of the subject on the augmented feedback (Salmoni 1984; Schmidt 1991; Schmidt et al. 1989). Important aspects of task-related information are ignored during feedback training such as environmental or task-inherent cues and intrinsic feedback, i.e., proprioception. However, concurrent feedback has been found to be effective for learning more complex tasks (Kovacs and Shea 2011; Marschall et al. 2007; Sigrist et al. 2013b; Snodgrass et al. 2010, Swinnen et al. 1997; Todorov et al. 1997; Wishart et al. 2002; Wulf et al. 1998, 1999). It is assumed that concurrent feedback facilitates the discovery of skills required for the novel task (Huegel and O'Malley 2010; Liebermann et al. 2002) and the understanding of the novel structure of the task (Braun et al. 2010; Wolpert and Flanagan 2010; Wolpert et al. 2011). Concurrent feedback can provide guidance through the complex movement and intuitively present the task requirements (Lüttgen and Heuer 2013; Sigrist et al. 2013a). Therefore, in this study, concurrent feedback is applied to enhance learning of a complex task.

Besides inducing a dependency, concurrent feedback can also force instantaneous corrections of task-irrelevant errors that appear randomly (Liu and Todorov 2007; Todorov 2004; Todorov and Jordan 2002; Wei and Körding 2009; Wolpert et al. 2011) and originate from noise in the motor system (van Beers 2009). Consequently, the taskrelevant parameters that determine the spatial and temporal features of the structure of the movement are not improved, as has been shown for complex trajectory learning (Sigrist et al. 2013b). In contrast to concurrent feedback, terminal summary feedback, which comprises feedback on a certain number of completed trials (Wulf and Shea 2002), was found to be effective (Schmidt et al. 1989) as it makes systematic, repeated errors apparent. Terminal feedback allows the subject to relate their own prior performance to their actual performance, which may enhance self-estimation. Self-estimation was found to be important for motor learning (Guadagnoli and Kohl 2001; Liu and Wrisberg 1997; Swinnen et al. 1990). Therefore, in this study, a trace of the performed movement is displayed in order to combine the benefits of concurrent and terminal feedback (Blandin et al. 2008; Sigrist et al. 2013a, b).

According to the specificity of learning hypothesis (Proteau 1992), the optimal source of sensory information is used to perform a movement. Commonly, augmented feedback provides reliable information to allow high performance during training. As a consequence, less reliable information that would also be available in conditions without augmented feedback such as kinesthetic information is surpassed. As a result, performance in non-feedback conditions drops as the most reliable information source of the feedback training is lacking. Ideally, feedback designs should therefore guide the subject toward the target movement without diminishing kinesthetic information processing (Robin et al. 2005; Wei and Körding 2009). Consequently, the linkage of landmarks or key features of the motor task to kinesthetic information should be emphasized in order to facilitate the recall in conditions without feedback (Sigrist et al. 2013a). Relatedly, the training conditions should match the testing condition (Ronsse et al. 2011). These requirements can be partly met by so-called bandwidth feedback (Ribeiro et al. 2011; Timmermans et al. 2009) since it is only displayed if the error exceeds a certain threshold. Therefore, in this study, a bandwidth feedback strategy is incorporated in the design of the visual feedback. This visual feedback is compared to multimodal feedback, i.e., visual feedback that was enhanced with auditory or haptic feedback.

Multimodal augmented feedback is believed to have several advantages over unimodal feedback. Information can be better processed if it is distributed to different modalities as people have different cognitive resources for information processing (Burke et al. 2006; Wickens 2002). Human senses are very specialized for different information. Spatial information is perceived more precisely through vision than audition (Freides 1974; Welch and Warren 1980) while audition is effective to perceive periodicity, regularity, and speed of motion (Kapur et al. 2005; Kramer 1994). Auditory feedback can help keep the focus on the task (Secoli et al. 2011) or guide the attention toward specific aspects 
of the movement (Schaffert et al. 2011). Haptic feedback is the only bidirectional modality (Hale and Stanney 2004; Minogue and Jones 2006), i.e., it can physically alter, disturb, or assist the movement of the subject. However, knowledge on the effects of multimodal feedback is limited, in particular, in complex motor task learning related to sports or rehabilitation (Sigrist et al. 2013a). On the one hand, studies on cross-modal integration suggest that multimodal feedback enhances perception (Seitz and Dinse 2007) and action (Carson and Kelso 2004), which may facilitate learning. On the other hand, as multimodal perception of the environment is critical to perform the task, augmented feedback provided in the same modalities may cognitively overload the learner or distract the learner from perceiving intrinsic feedback (Sigrist et al. 2013a). Therefore, in this study, unimodal visual feedback, which is the most common form of augmented feedback, is compared with the combination of visual feedback with auditory feedback (in the form of movement sonification), and with haptic feedback in order to exploit modality-specific advantages (Huang et al. 2005; Welch and Warren 1980).

Movement sonification represents the mapping of a measured kinematic or kinetic variable to an audio variable such as amplitude or frequency. When the value of a variable changes, the sound is altered according to the underlying mapping function. Movement sonification has already been demonstrated to enhance the perception (Effenberg 2005; Schmitz et al. 2013); performance (Schaffert et al. 2011), and learning of dynamic/temporal aspects of a movement (Chollet et al. 1992). This enhancement is based on audio-motor coupling (Brown and Palmer 2012), i.e., the auditory and motor systems are co-activated when a movement and its related sound is produced, even if only one system is excited (Kim et al. 2008; Schmitz et al. 2013; Seitz et al. 2006; Shams and Seitz 2008; Zatorre et al. 2007). It is assumed that motor imagery may lead to coactivation of auditory and motor brain regions, especially if an association between movement and sound is well learned (Zatorre et al. 2007). Vice versa, imagination of the sound associated with a movement, i.e., the movement sonification, may facilitate the recall of the movement (Ronsse et al. 2011; Sigrist et al. 2013a) as auditory imagery is believed to contribute to motor planning (Hubbard 2013). Therefore, in this study, it is tested whether the addition of movement sonification to visual feedback enhances retention of dynamic aspects more than visual feedback alone.

Haptic feedback has often been applied to guide through correct movements, allowing different grades of freedom in the movement [see (Marchal-Crespo and Reinkensmeyer 2009; Sigrist et al. 2013a) for reviews]. However, completely haptically guided movements can make subjects passive (Israel et al. 2006) and cause them to rely on the provided assistance (Marchal-Crespo and Reinkensmeyer 2008a; Reinkensmeyer et al. 2009). An alternative approach to haptic guidance is the application of error or movement amplification (Chen and Agrawal 2013; Huang and Patton 2013; Patton etal. 2006). As learning is error-driven (Thoroughman and Shadmehr 2000), an optimal amplification of the perceived movement error accelerates the learning process (Cesqui et al. 2008; Emken and Reinkensmeyer 2005; Milot et al. 2010; Patton et al. 2006, 2013). Furthermore, motor learning should force the internalization of proprioceptive information of correct movements (Chiviacowsky and Wulf 2007; Winstein 1991). Therefore, for this study, a reactive haptic feedback is designed that emphasizes the perception of the error without amplifying it. The movement is hindered by generating an increasing counterforce when the spatial deviation from the trajectory is increased. The counterforce is not applied when the movement was directed toward the trajectory. This reactive haptic feedback design was inspired by a "path of least resistance" concept, and the design was assumed to prevent passiveness and thus increase the involvement of the subject in the training process (Wulf 2007). It is supposed that the haptic reactive feedback can force the subject to remember how counterforces can be avoided in the next movement trial, i.e., to effectively adjust the structure of the task (Braun et al. 2010; Wolpert and Flanagan 2010; Wolpert et al. 2011). Thus, in this study, haptic feedback is added to enhance proprioception, involvement, and correction of the accentuated movement error.

So far, investigations on augmented feedback have mostly focused on tasks that were rather simple, appear artificial, lacked interaction with a realistic environment, or involved only a low number of muscles and limbs (Sigrist et al. 2013a). Since the transfer of conclusions derived from studies on simple, artificial task to complex task learning in sports and rehabilitation is limited (Guadagnoli and Lee 2004; Winstein 1991; Wulf and Shea 2002), it is worth investigating augmented feedback in complex tasks (Krakauer and Mazzoni 2011; Sigrist et al. 2013a; Wolpert et al. 2011). Therefore, a rowing simulator was developed that features rendering of a realistic landscape, soundscape, and water resistance at the blade. The realistic multimodal interactions in the simulator enable the investigation of real rowing in a controlled environment (Rauter et al. 2010, 2013, von Zitzewitz et al. 2008). In this study, the simulator was used to investigate the effectiveness of augmented visual, audiovisual, and visuohaptic feedback that was provided in addition to the rendered scenario with regard to learning a complex task related to sports. As all three feedback designs included the same visual feedback, the benefit of additional auditory or haptic feedback can be determined. The investigated task, i.e., trunk-arm rowing, has a high functional complexity (Guadagnoli and Lee 2004) as the subjects 
were naïve and the movement is multidimensional and incorporates a complex velocity profile. In a common rowing training situation, a coach teaches the athlete a certain rowing technique. Thereby, the coach has a certain textbook technique in mind that includes a textbook oar movement, i.e., a reference oar movement. This textbook technique is commonly agreed on to lead to the fastest boat propulsion. This is especially of importance for a group sports like rowing, where all athletes have to use the same technique in order to minimize interference with each other while maximizing boat propulsion. Without the augmented (e.g., verbal) feedback of the coach, an athlete would not be able to learn this textbook technique. Thus, in this study, instead of training with a coach, a reference oar movement was taught to the subjects through augmented concurrent feedback provided by visual, auditory, and haptic displays.

Augmented feedback was applied during training sessions in addition to the rendered scenario, i.e., in addition to the intrinsic feedback. The augmented visual, auditory (sonification), and haptic feedback disappeared for the most part when the movement was performed correctly. Consequently, when the movement was performed correctly in the feedback conditions, the subject perceived only the rendered scenario, as in non-feedback conditions. The visual feedback aimed at forcing a focus on task-relevant aspects while minimizing a dependency on the feedback. Therefore, transfer of mastered skills to conditions without feedback, i.e., learning, was hypothesized. The audiovisual feedback consisted of the visual feedback and a sonification of the oar movement. Audiovisual feedback is hypothesized to boost learning of the velocity profile compared to visual feedback alone, as the sonification is expected to be remembered in non-feedback conditions. For the visuohaptic feedback, a reactive force that depended on the deviation from the target path was applied on top of the rendered water resistance. The reactive force was expected to enforce involvement and to direct the focus on proprioception. Thus, the spatial aspects of the movement were hypothesized to be learnt better with visuohaptic feedback than with visual feedback only. However, task-inherent forces, i.e., the rendered water resistance, can alter the perception of the forces of the augmented haptic feedback (MarchalCrespo and Reinkensmeyer 2008a). Therefore, the effects of the haptic feedback on learning the movement during the phase when the blade was in the water were expected to be smaller than outside the water. The multimodal feedback designs did not contain additional information about the performance in comparison with the visual feedback. However, the additional auditory feedback was intended to facilitate perception of temporal aspects and the additional haptic feedback of spatial aspects of the movement. In general, it was hypothesized that multimodal feedback is more

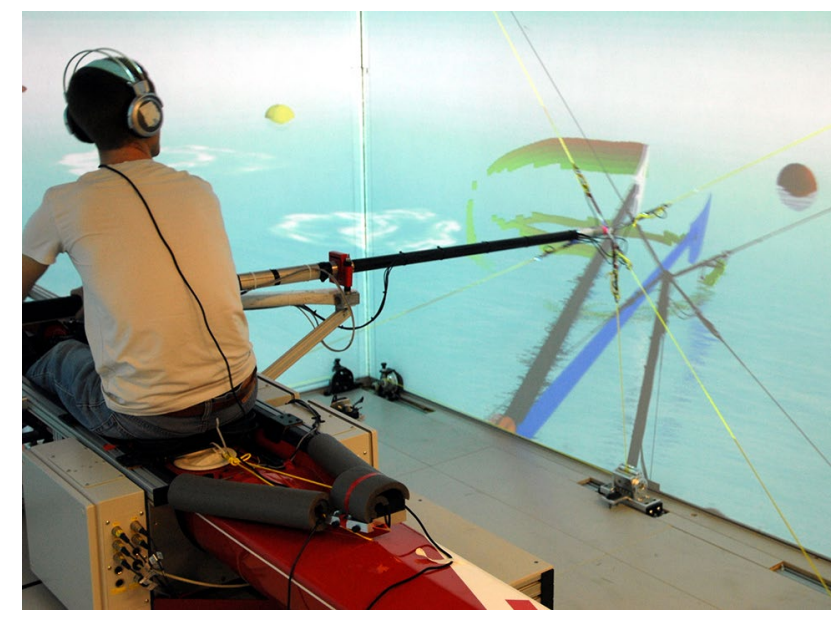

Fig. 1 System setup

effective than unimodal visual feedback to learn the complex movement due to intersensory facilitation and exploitation of modality-specific advantages.

\section{Methods}

Subjects

A total of 24 subjects (7 females, 17 males, 21-33 years, mean age 26.1 years, SD 3.0 years) were recruited, mainly from the university (students). The subjects were healthy, had normal hearing and normal or corrected-to-normal vision. All subjects had no prior experience with the task and confirmed to be non-rowers and to do at least half an hour of sport per week. They were randomly assigned to one of three training groups including eight participants each, i.e., either to the visual feedback group (V; 2 females, 6 males, 21-33 years, mean age 26.1 years, SD 4.4 years), audiovisual feedback group (VA; 3 females, 5 males, 22-30 years, mean age 26.8 years, SD 2.9 years), or the visuohaptic feedback group (VH; 2 females, 6 males, 24-28 years, mean age 25.4 years, SD 1.3 years). The subjects signed an agreement following the guidelines of the local ethics commission, which had approved the study.

Apparatus and task

In our laboratory, a realistic rowing simulator utilizing a tendon-based parallel robot has been developed (Fig. 1) (Rauter et al. 2011). A real but trimmed rowing boat was placed in the middle of a Cave Automated Virtual Environment (CAVE). A visual ocean scenario and augmented visual feedback was programmed (Unity) and projected on three screens $(4.4 \mathrm{~m} \times 3.3 \mathrm{~m}$, projectors: Projection Design 

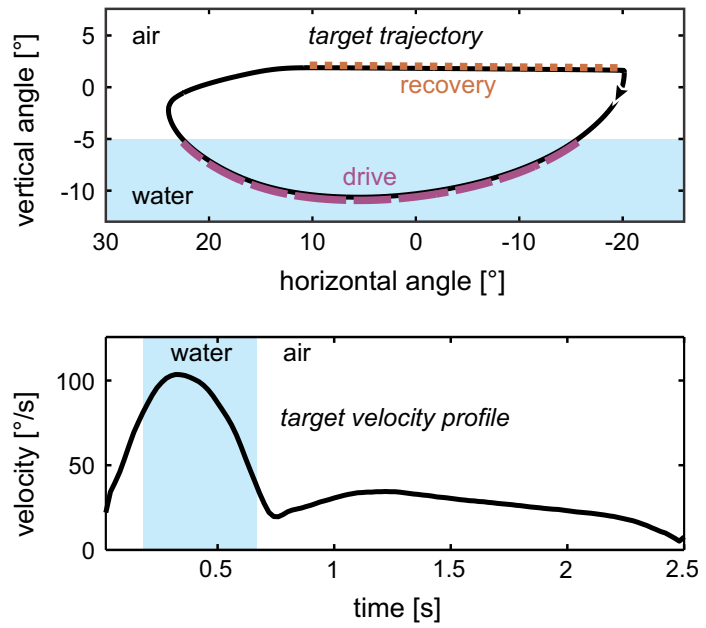

Fig. 2 Target oar movement to be learned. Top target trajectory (black) with the illustration of drive phase (orange dotted) and recovery phase (magenta dashed). Bottom target velocity profile of the oar movement

F3+, Norway) surrounding the boat in U-form (update rate always $>30 \mathrm{fps}$ ). Auditory rendering of the oar-water interaction and movement sonification $(\mathrm{C}++)$ was updated at $\sim 30 \mathrm{~Hz}$ and displayed through standard stereo headphones (frequency range: $14 \mathrm{~Hz}$ to $26 \mathrm{kHz}$ ). Haptic simulation of the water resistance and haptic augmented feedback was implemented on a tendon-based parallel robot with a MATLAB/Simulink ${ }^{\circledR}$ model running on an XPC target at $1,000 \mathrm{~Hz}$. In this study, portside sweep rowing was simulated, i.e., the subject manipulated a single oar with both hands. This trimmed oar was connected to the five ropes of the rope robot at the outer end and virtually elongated on the screens. The horizontal and vertical oar angles were calculated based on the end-effector positions. By moving the oars through the rendered water, a subject was able to accelerate and decelerate the boat in the virtual environment.

Subjects were asked to learn a complex oar movement by using their trunk and arms. Such so-called trunk-arm rowing is often used as a warm-up exercise and also as a technical exercise in rowing trainings. The target rowing cycle in trunk-arm rowing consists of two phases with particularly different challenges (Fig. 2). In the drive phase, the blade is in the water. Effort is needed to pull the blade through the water quickly, whereby the blade is meant to be completely covered by water just at its deepest point. During the recovery phase, the blade is outside the water. The movement is slower and less effort is required compared to the drive phase. However, the straight horizontal blade movement at constant height is challenging to coordinate with the trunk and arms (Fig. 2, top). The combination of the fast drive phase and the slow recovery phase results in a complex oar velocity profile that is difficult to perform, particularly because of the change of media, i.e., water and air (Fig. 2, bottom). Blade rotation was not included. The target movement was prerecorded from an expert rower. In post-processing, the trajectory was smoothed in space and velocity to form a cyclic $C^{2}$ continuous trajectory, i.e., a cyclic trajectory that can be continuously differentiated two times. The trajectory was adapted to a movement range at the oar handle center of $0.67 \mathrm{~m}$ $\left(\hat{=} 44^{\circ}\right)$ horizontally and $0.19 \mathrm{~m}\left(\hat{=} 12.5^{\circ}\right)$ vertically that could be performed by subjects taller than $\sim 1.65 \mathrm{~m}$ (inclusion criteria). The target movement was fit to a typical training stroke rate of 24 strokes per minute. During the whole study, the seat was fixed at a position where the subject's legs were extended.

\section{Augmented feedback designs}

For the visual feedback, a blue, virtual oar showed the target movement on the screen on portside at the target position and at the target velocity (Fig. 1). The transparency of the blue oar was linearly mapped onto the angular distance to the own oar $\left(0^{\circ}\right.$ deviation: $100 \%$ transparent; lhorizontal deviationl + |vertical deviationl $>57.3^{\circ}$ deviation: $0 \%$ transparent, whereby the blue oar was clearly visible on the screen at a deviation greater than $4^{\circ}$ ). Additionally, a terminal feedback in form of a trace was drawn at locations where any part of the blade deviated more than $3.6^{\circ}$ vertically or $1.9^{\circ}$ horizontally from the target trajectory depicted in Fig. 2, top (nearest neighbor calculation; the values differ because the blade is higher than it is wide). The trace was built with single 3D-markers (see Fig. 1), which desaturated linearly until they disappeared after $8 \mathrm{~s}$ ( $>3$ target cycles). Moreover, the larger the deviation of the 3D-markers from the trajectory, the less green they were and the more reddish they appeared (deviations $>11.5^{\circ}$ : completely red). In other words, the more the own oar deviated from the blue target blade and the more the oar deviated from the trajectory, the less transparent the blue target oar appeared and the more the trace was drawn. Vice versa, if the oar was moved exactly synchronous with the blue target oar and on the trajectory, the subject could only see the own oar moving in the scenario.

The auditory feedback was based on the sonification of the oar movement. The horizontal oar angle was mapped linearly with a change of frequency when the oar was outside the water. The frequency of a violin sound of the target movement ranged from 54.5 to $91.58 \mathrm{~Hz}$, i.e., approximately A (A1) to Fis (F\#2). A purling sound was played when the blade was in the water. In the transition phase from air to water and vice versa, the amplitude of the violin or purling water sound was faded in or out, respectively. Subjects were asked to synchronize the own movement 
displayed on the right headphone to the sonified reference oar movement displayed on the left headphone. In conditions without feedback, only the purling sound of the own oar was rendered. Even though the oar angles were used as an input for the mapping function, the sonification was expected to represent the dynamic aspects of the oar movement as changes of the oar angles were dominantly perceived.

The haptic reactive feedback was based on an elastic torque field $\left(\tau_{\text {elast }}\right)$ of a path controller. The elastic torque field for the path controller was calculated in real time from the negative gradient of a potential field. The current magnitude of the potential field increased with the distance to the path, i.e., to the target trajectory [see (Rauter et al. 2011) for a more detailed description of the elastic torque field]. The controller applied resistive torques against the end-effector movement when the end-effector moved away from the target trajectory (Eq. 1, i). A dead zone was defined around the target trajectory, i.e., no resistive forces were perceivable for deviations smaller than $\sim 1^{\circ}$. Consequently, within the dead zone, the subject felt the normal water resistance when the blade was in the water and no resistance when the blade was in the air. When the end-effector approached the target trajectory, an elastic torque field pushed the end-effector toward the target trajectory (Eq. 1, ii):

$\tau_{\text {react }}=\left\{\begin{array}{llll}-\frac{\left\|\tau_{\text {elast }}\right\|}{\|v\|} v, & \text { if }\left(v^{T} \tau_{\text {elast }}<0\right. & \& & \|v\|>0) \\ \tau_{\text {elast }}, & \text { else } & \text { (i) }\end{array}\right.$

The parameters of the feedback designs (e.g., deviation threshold for trace, mapping function of sonification, gains of the haptic reactive feedback) were set based on the best knowledge gained from a systematic evaluation of feedback in pilot studies and prior studies (Sigrist et al. 2011, 2013b).

\section{Experimental protocol}

Subjects were asked to come to the laboratory on three consecutive days. On Day 1, following general instruction, one investigator explained the handling and safety features of the simulator to the subject.

The instruction of the target movement to the subject before the baseline test was done with robotic guidance, i.e., a position controller moved the oar through the target trajectory with the target velocity profile. The position controller comprised a proportional component $\left(P_{\text {pos }}=6000 \mathrm{Nm} / \mathrm{rad}\right)$ and a derivative component $\left(D_{\text {pos }}=170 \mathrm{Nms} / \mathrm{rad}\right)$ that corrected for position and velocity errors between the measured and target horizontal and vertical oar angles and velocities at the end-effector, i.e., at the end of the trimmed oar.

As a first instruction, the investigator sat in the boat and demonstrated the movement to the subject. Thereby, the robot guided the movement with the position controller for $\sim 30 \mathrm{~s}$. This primary instruction was meant to familiarize the subject with movement range and velocity, i.e., to prevent the subject to be taken by surprise. As a second instruction, the subject sat in the boat, held the oar with both hands, and experienced the robot-guided oar movement for $180 \mathrm{~s}(\hat{=} 72$ target cycles). The subject was asked to learn the movement since they would be requested to reproduce the movement trajectory and velocity profile in a subsequent baseline test. Subjects were not instructed on how to use the robot-guided exemplary movement, i.e., on being active or passive or on what to focus. During the instruction, the visual scenario with the virtually elongated oar of the subject and the purling sound of the blade in the water were rendered.

In the following baseline test of $180 \mathrm{~s}$ ( $\hat{=} 72$ target cycles), the subjects rowed without robotic guidance. Thus, the subjects were asked to perform the movement by remembering the instruction, i.e., they relied on intrinsic feedback. As in all subsequent tests or training sessions, the visual scenario, the purling sound of the own oar on the right headphone, and the water resistance were rendered. After the baseline test, the subject was instructed about the visual, audiovisual, or visuohaptic augmented feedback, depending on the subject's group adherence. The subject could familiarize with the feedback for $\sim 60 \mathrm{~s}$ by moving the oar. Five training sessions followed. One training session consisted of training that was enhanced with augmented feedback (feedback training) lasting $180 \mathrm{~s} \mathrm{(} \triangleq$ back (non-feedback trials) lasting $60 \mathrm{~s} \mathrm{( \bumpeq} 24$ target cycles). A break of $\sim 25 \mathrm{~s}$ was included between feedback training and non-feedback trials. Day 2 started with a retention test of $180 \mathrm{~s}$, which was identical to the baseline test, followed again by five training sessions. On Day 3, another retention test of $180 \mathrm{~s}$ was conducted. A long-term retention test was not included as in a previous study on a related rowing-type task, no significant differences between a one-day retention test and a seven-day retention test were found (Sigrist et al. 2013b). Thereafter, subjects were asked to complete a short questionnaire about feedback comfort, practicability, level of activity, and their learning strategy.

In the baseline test, retention tests, and non-feedback trials, the subjects were verbally asked to increase or decrease the stroke rate, if they left the range of 22-26 strokes/ $\mathrm{min}$ in order to avoid effects on performance caused by a speed-accuracy trade-off. There were no restrictions on the stroke rate during the feedback training.

Data analysis

\section{Kinematic data}

Kinematic data, i.e., the vertical and horizontal oar angles measured by the robot at $100 \mathrm{~Hz}$, were analyzed 

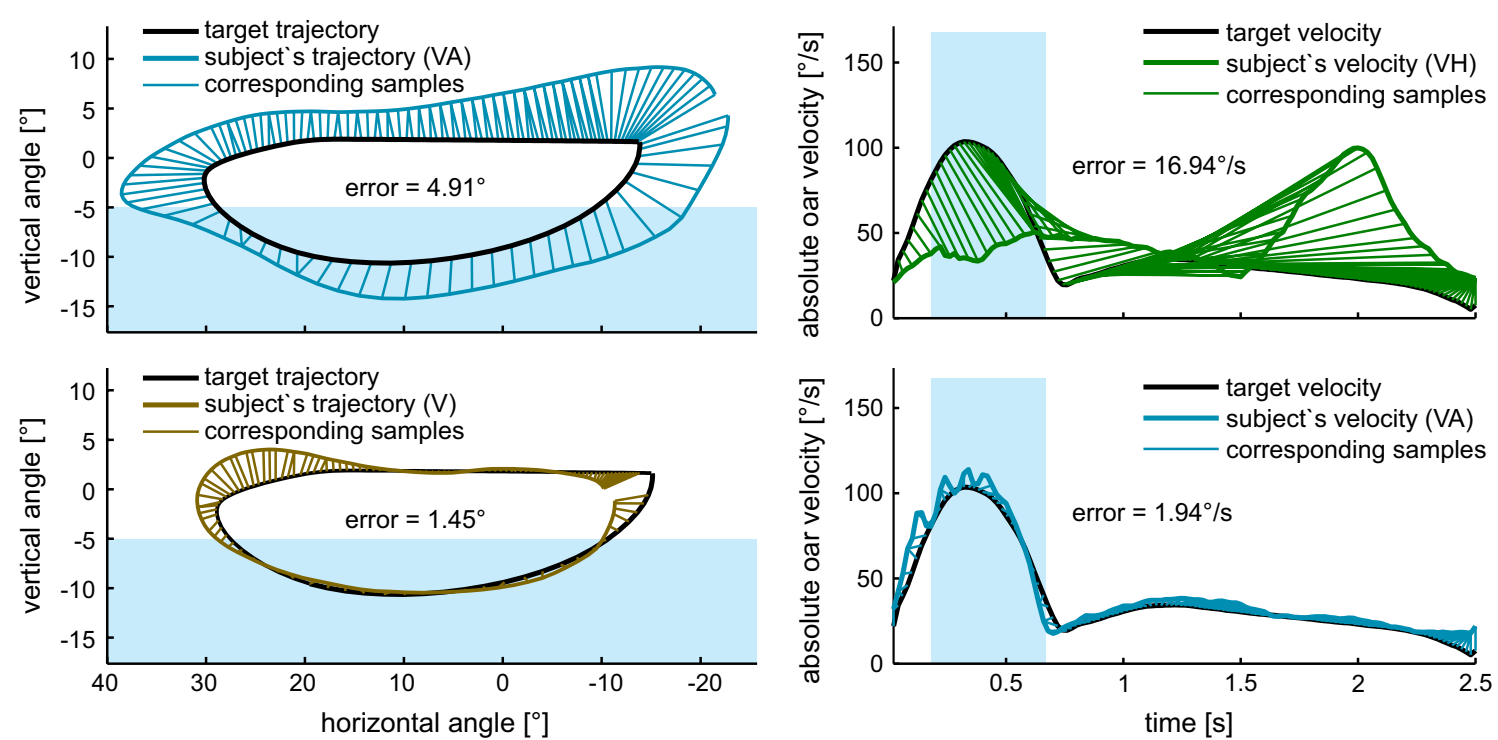

Fig. 3 The overall spatial error (left column) and velocity error (right column) of different exemplary cycles using DTW are illustrated. The exemplary cycles are chosen from the subjects with the highest mean

in custom-written programs in MATLAB (MathWorks, Natick, Massachusetts, USA). The first five cycles and the last cycle of each condition, i.e., of the baseline test, feedback trainings, non-feedback trails, and retention tests were excluded from the analysis in order to avoid transition effects (subjects needed time to accelerate to a steady state). Cycles beyond 22-26 strokes/min were excluded from the baseline test, retention tests, and non-feedback trials (V: 19.81, VA: 18.00 , VH: $18.75 \%$ of all cycles excluded). During the feedback training sessions, from time to time, the subjects interrupted or shortcut the movement to synchronize with the target movement. Therefore, for the feedback training, cycles below 18 strokes/min and above 30 strokes/min were excluded (V: 0.02, VA: 0.18, VH: $0.11 \%$ of all cycles excluded). The data of the last two non-feedback trials and the last feedback training of one subject of VH were lost.

Measured data were divided into cycles (rowing strokes). Cycles of the feedback training were thereby divided at the point in time corresponding to the minimal horizontal angle of the target movement. In the conditions without feedback, cycles were divided at the point in time corresponding to the minimal horizontal angle of the subject's movement, as an online target movement was missing. The cycles were resampled to 125 data points $(50 \mathrm{~Hz})$ and compared to the target cycle consisting of 125 data points.

Different variables were extracted from the oar angles to evaluate the subjects' spatial and temporal performance. To extract an overall spatial error, the measured oar movement of the subject was compared to the target oar movement by using a spatiotemporal analysis with dynamic time warping error in the baseline test (top row) and smallest mean error in the retention test on Day 3 (bottom row)

(DTW) (Giese and Poggio 2000). Time warping avoids overestimation of errors arising from small temporal shifts between the measured and target movements in contrast to calculating the error sample by sample, i.e., between samples with the same index (Vlachos et al. 2003). To obtain sample correspondence, DTW provides the possibility of weighting the impact of temporal shifts and spatial errors (distance between corresponding samples). In this study, the weighting of the temporal shifts was set to zero, i.e., the cost function minimized the spatial errors of the subject's performance considering the sequential order of the measured data points (Giese and Poggio 2000). Therefore, the spatial error could be calculated while the continuity of the movement (causal temporal order of samples) was assured. The spatial error extracted with DTW was used to describe the spatial error of entire rowing cycles, i.e., the overall spatial error (Fig. 3, left column). The trajectory had two distinct phases, i.e., the drive phase and the recovery phase (Fig. 2, top). The spatial error was calculated by applying DTW analysis also in these two phases separately in order to get a more detailed insight. To extract a temporal error (the velocity error), DTW was used analogously: Deviations between the absolute value of the measured and the target oar angle velocity profile were calculated (Fig. 3, right column).

\section{Questionnaire}

The questionnaire answers given by the subjects after the retention test on Day 3 assessed two aspects. Firstly, they could rate the comfort to train with the feedback, how 
useful it was for learning the task and how active they were in using the feedback. The answer scale ranged from 1 (not at all) to 7 (very much). Secondly, on Day 3, subjects were asked about their strategy for recalling the movement and could choose one answer out of seven: (1) demonstration of Day 1, (2) landmarks in the virtual reality (VR), (3) real landmarks, (4) sounds in the VR, (5) real sounds, (6) their feeling, or (7) other.

\section{Statistical analysis}

Statistical analysis was performed with IBM PASW Statistics 20 (IBM Corp., New York City, USA). For each cycle, mean absolute errors were calculated for each variable, i.e., overall spatial error, spatial error drive phase, spatial error recovery phase, and velocity error. A mean value of each variable was then calculated for each condition, i.e., baseline test, retention test, feedback training, or non-feedback trial. The subjects' mean values of each variable were used as an input for the statistical analysis, resulting in 24 mean values of each variable in each condition (three groups of eight subjects).

One-way ANOVA was used to assess whether groups differed significantly at the baseline test on Day 1 in any variable. Tukey HSD post hoc tests were used for multiple comparisons.

A linear mixed-model design was used to compare performance between groups during feedback training and non-feedback trials. The group was set as the fixed factor and the two training days and five repetitions on each day as the random factors. Multiple comparisons between groups were performed with Tukey HSD post hoc tests. A mixed-model design was also used to analyze performance during training with feedback and the non-feedback trials within each group. Feedback on/off (feedback training or non-feedback trial) was set as the fixed factor and the two training days and five repetitions on each day as the random factors.

Repeated-measures ANOVA was used on the baseline and retention tests to assess interaction ( 3 groups $\times 3$ tests) and between group effects ( 3 groups). Follow-up repeatedmeasures ANOVA was also used to assess learning within each group. Post hoc Bonferroni test was applied for multiple comparisons between the tests. Violations of sphericity were corrected using Greenhouse-Geisser correction.

One-way ANOVA was also used to assess whether the groups differed in the learning rate $R$, i.e., the improvements from baseline test on Day 1 to the retention test on Day 2 ( $R_{\mathrm{bl1}}$ to re2 $)$, from baseline on Day 1 to the retention test on Day 3 ( $R_{\mathrm{b} 11}$ to re3 $)$, and from the retention test on Day 2 to the retention test on Day 3( $R_{\mathrm{re} 2}$ to re3). The learning rate was normalized by the skill level before the training sessions.
Group differences in the questionnaire answers on the comfort and usability of the feedback designs and on its active usage were analyzed with a Kruskal-Wallis test. Group effects on the questionnaire answers on what subjects' recalled in order to perform the movement were evaluated with a Chi-square test. Two-sided $p$ values below 0.05 were considered to indicate significance, and $p$ values below 0.1 to indicate tendencies in all statistical tests.

\section{Results}

One-way ANOVA showed no significant difference between groups in any variable at the baseline test.

\section{Training sessions}

For training with feedback, the univariate mixed-model ANOVA resulted in significant main effects of group in all variables, except spatial error in drive phase. Post hoc Tukey HSD tests revealed significantly smaller overall spatial error for $\mathrm{VH}$ than $\mathrm{V}$ and significantly smaller spatial error during recovery for VA and $\mathrm{VH}$ than for V. Velocity error significantly differed between all groups, whereby VA showed the smallest and VH the highest error (Fig. 4; Table 1).

In non-feedback trials, main group effects were found for overall spatial error and velocity error. Spatial error was significantly smaller for $\mathrm{V}$ than for $\mathrm{VH}$. Velocity error was significantly smaller in V and VA compared to VH (Fig. 4; Table 1).

All groups had significantly lower overall spatial errors and spatial errors during recovery in the feedback training than in non-feedback trials. Furthermore, VH had also lower errors during the drive phase in the feedback training than in the non-feedback trials. In $\mathrm{V}$ and $\mathrm{VH}$, the velocity error was higher in training with feedback than in the nonfeedback trials (Fig. 4; Table 1).

Baseline to the retention tests

Repeated-measures ANOVA on the baseline test, retention test on Day 2, and retention test on Day 3 revealed no significant interaction of group $\times$ test and no main effect for group in any variable. However, the main effects of test (baseline test, retention test on Day 2 and on Day 3) were significant for all variables, showing a decrease in the errors along the tests. For V, follow-up repeated-measures ANOVA within the group revealed significant main effects for velocity error and tendencies for spatial error during drive and for overall spatial error. Within VA, a significant main effect was found in all variables, i.e., a decrease in the spatial errors and velocity 
Fig. 4 Mean overall spatial error (top) and mean velocity error (bottom) of the oar movement. Group mean values are represented with symbols and connected with lines ( $V$ yellow circle, $V A$ blue diamond, $\mathrm{VH}$ green square). Group standard deviations are indicated with vertical lines. White background indicates baseline $(b l)$ and retention tests (re), dark gray the training with feedback $(f b)$, and fair gray the non-feedback trials $(n)$
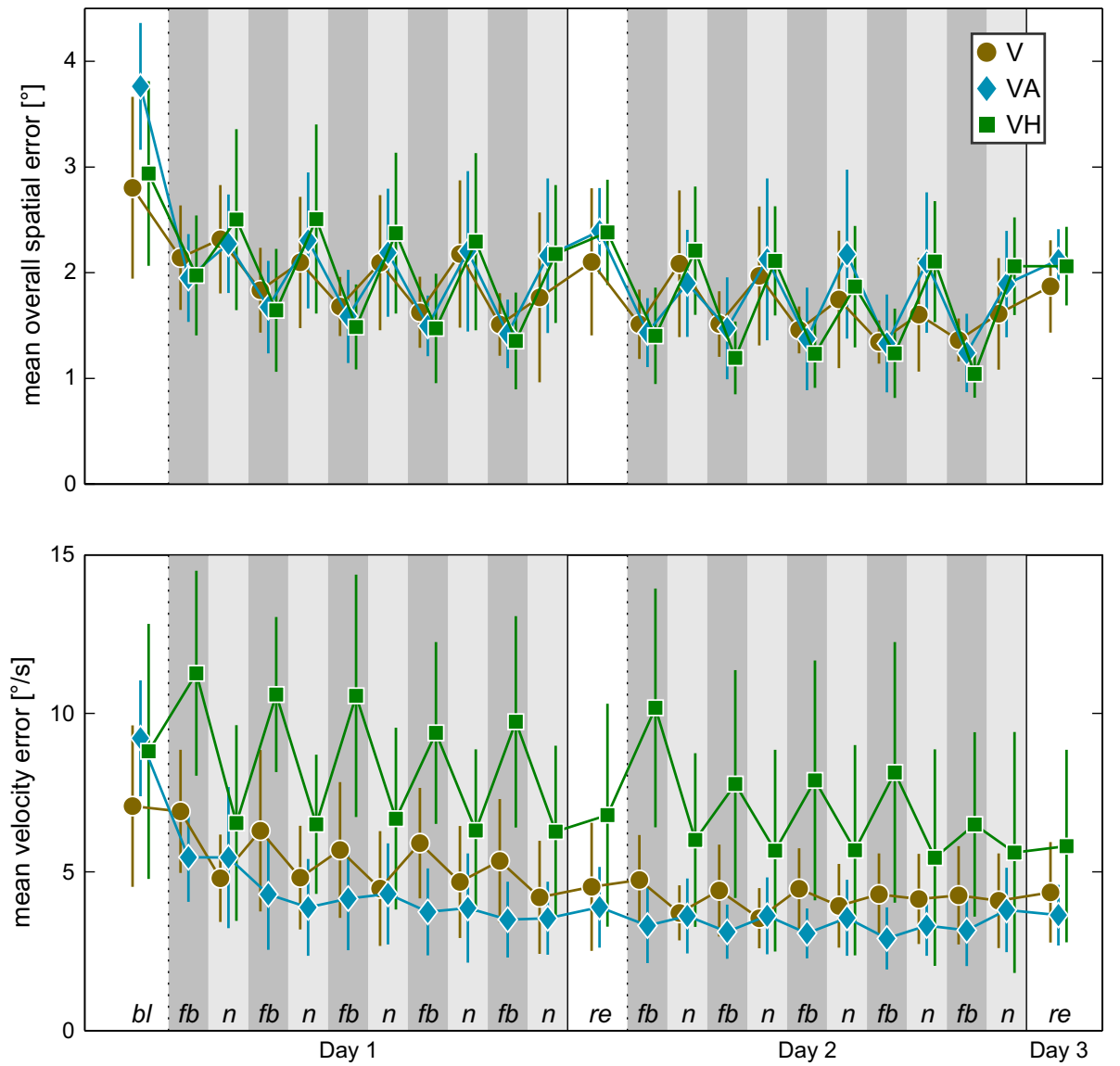

Table 1 Statistically significant results $(p<.05)$ of the training sessions applying a linear mixed-model design with Tukey HSD post hoc tests

\begin{tabular}{|c|c|c|c|c|c|c|c|c|c|}
\hline \multirow[t]{3}{*}{ Variable } & \multicolumn{6}{|c|}{ Between group differences } & \multirow{2}{*}{\multicolumn{3}{|c|}{$\frac{\text { Within-group differences }}{\text { Feedback to non-feedback trials }}$}} \\
\hline & \multicolumn{3}{|l|}{ Feedback training } & \multicolumn{3}{|c|}{ Non-feedback trials } & & & \\
\hline & $\begin{array}{l}\text { Main effect } \\
\text { post hoc }\end{array}$ & $p$ & $\eta_{p}^{2}$ & $\begin{array}{l}\text { Main effect } \\
\text { post hoc }\end{array}$ & $p$ & $\eta_{p}^{2}$ & Main effect & $p$ & $\eta_{p}^{2}$ \\
\hline \multirow{2}{*}{$\begin{array}{l}\text { Spatial error } \\
\text { overall }\end{array}$} & $F_{(2,227)}=4.04$ & .012 & .038 & $F_{(2,226)}=3.24$ & .041 & .028 & \multicolumn{3}{|l|}{ Lower with feedback: } \\
\hline & VH lower V & .010 & & V lower VH & .032 & & $\begin{array}{l}\text { V: } F_{(1,149)}=17.38 \\
\text { VA: } F_{(1,149)}=49.45 \\
\text { VH: } F_{(1,146)}=70.67\end{array}$ & $\begin{array}{l}<.001 \\
<.001 \\
<.001\end{array}$ & $\begin{array}{l}.105 \\
.249 \\
.326\end{array}$ \\
\hline \multirow[t]{2}{*}{ Spatial error drive } & & & & & & & \multicolumn{3}{|l|}{ Lower with feedback } \\
\hline & & & & & & & VH: $F_{(1,146)}=6.17$ & .014 & .041 \\
\hline \multirow{4}{*}{$\begin{array}{l}\text { Spatial error } \\
\text { recovery }\end{array}$} & $F_{(2,227)}=15.33$ & $<.001$ & .119 & & & & \multicolumn{3}{|l|}{ Lower with feedback } \\
\hline & VA, VH lower V & $<.001$ & & & & & $V: F_{(1,149)}=21.14$ & $<.001$ & .117 \\
\hline & & & & & & & VA: $F_{(1,149)}=41.95$ & $<.001$ & .220 \\
\hline & & & & & & & VH: $F_{(1,146)}=42.46$ & $<.001$ & .225 \\
\hline \multirow[t]{3}{*}{ Velocity error } & $F_{(2,227)}=108.83$ & $<.001$ & .490 & $F_{(2,226)}=23.12$ & $<.001$ & .170 & \multicolumn{3}{|l|}{ Higher with feedback } \\
\hline & VA lower V, VH & $<.001$ & & V, VA lower VH & $<.001$ & & $V: F_{(1,149)}=13.46$ & $<.001$ & .083 \\
\hline & V lower VH & $<.001$ & & & & & VH: $F_{(1,146)}=34.07$ & $<.001$ & .189 \\
\hline
\end{tabular}

error over the tests. VH significantly decreased errors in all variables except spatial error during drive (Fig. 5; Table 2).
One-way ANOVA on the learning rate found a main effect of group for velocity error from the baseline test on Day 1 to the retention test on Day $2\left(F_{(2,21)}=4.31\right.$, 

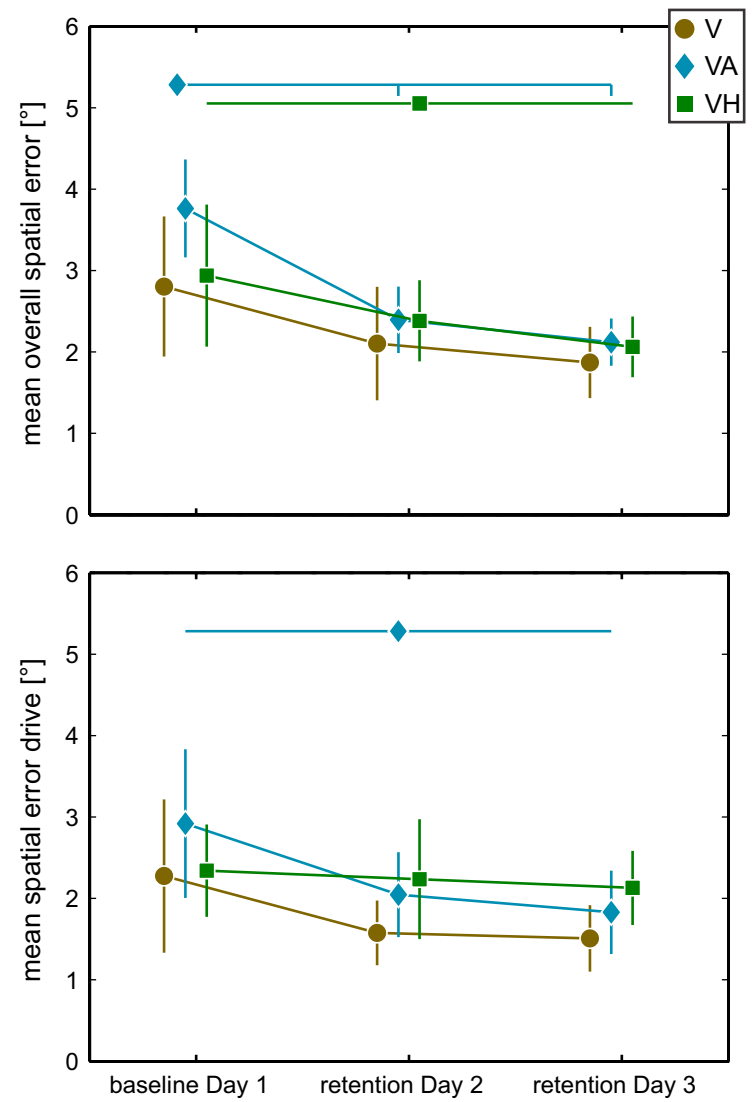

Fig. 5 Learning curves of all extracted variables (errors) from baseline on Day 1 to the retention tests on Day 2 and Day 3. Group mean values are represented with symbols and connected with lines $(V$ yellow circle, VA blue diamond, VH green square). Group standard deviations are indicated with vertical lines. Horizontal lines indicate significant within-group effects of learning over the tests revealed from the repeated-measures ANOVA. Additional vertical marks indi-

$\left.p=.027, \eta_{p}^{2}=.291\right)$. Tukey HSD post hoc tests revealed a significantly higher learning rate for VA compared to $\mathrm{VH}$ ( $p=.022$ ). A main effect for velocity error was also found from baseline test on Day 1 to the retention test on Day 3 $\left(F_{(2,21)}=4.13, p=.031, \eta_{p}^{2}=.282\right)$. Again, Tukey HSD post hoc tests revealed a significantly higher learning rate for VA compared to VH ( $p=.034)$. No further differences of learning rate between the groups were found (Fig. 5).

\section{Questionnaire answers of the subjects}

On average, the visuohaptic feedback was rated as less comfortable than the visual and audiovisual feedback. All groups rated their feedback to be similarly useful, and they were similarly active in using the feedback. In the retention test on Day 3, 5 of 8 subjects of VA relied on their feeling to recall the movements. $\mathrm{V}$ and $\mathrm{VH}$ relied more on visual landmarks in the VR scenario (Fig. 6). Statistical analysis did not reveal any significant group effects on the questionnaire answers.
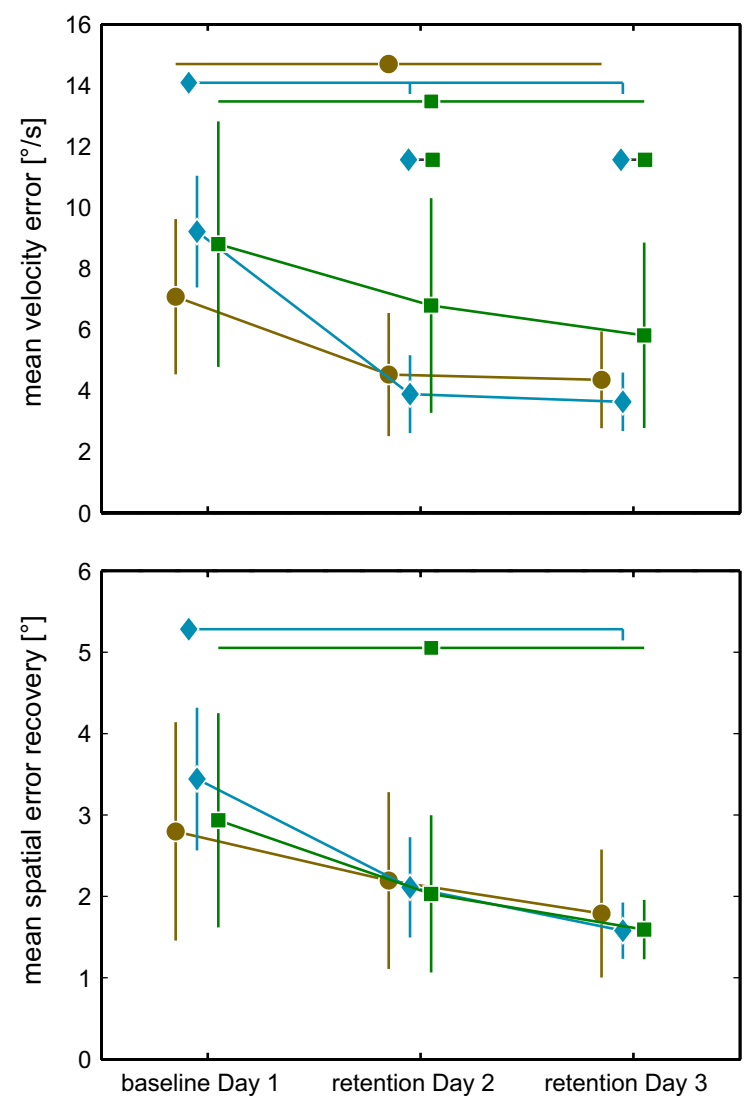

cate significant differences from baseline to a retention test based on Bonferroni-corrected post hoc tests. Significant differences between groups in terms of skill level-corrected learning rate from baseline to a retention tests revealed from the one-way ANOVA are indicated by black horizontal lines limited by the according group symbols at the respective retention test

\section{Discussion}

The augmented feedback designs used in this study were expected to facilitate complex motor learning in rowing. As hypothesized, the training with unimodal visual feedback was effective, shown by a tendency in the reduction of the overall spatial error and by a significant reduction of the velocity error from the baseline test to the retention tests. The addition of auditory feedback was expected to boost learning of the velocity profile since sonification mediates dynamic aspects of a movement. Additional haptic feedback was hypothesized to foster learning of spatial aspects due to enhanced perception of spatial errors and due to increased involvement. Both multimodal feedbacks significantly reduced the overall spatial error and velocity error in retention tests. However, as visual feedback alone was already effective, the benefits of additional sonification or haptic reactive feedback were more subtle. 


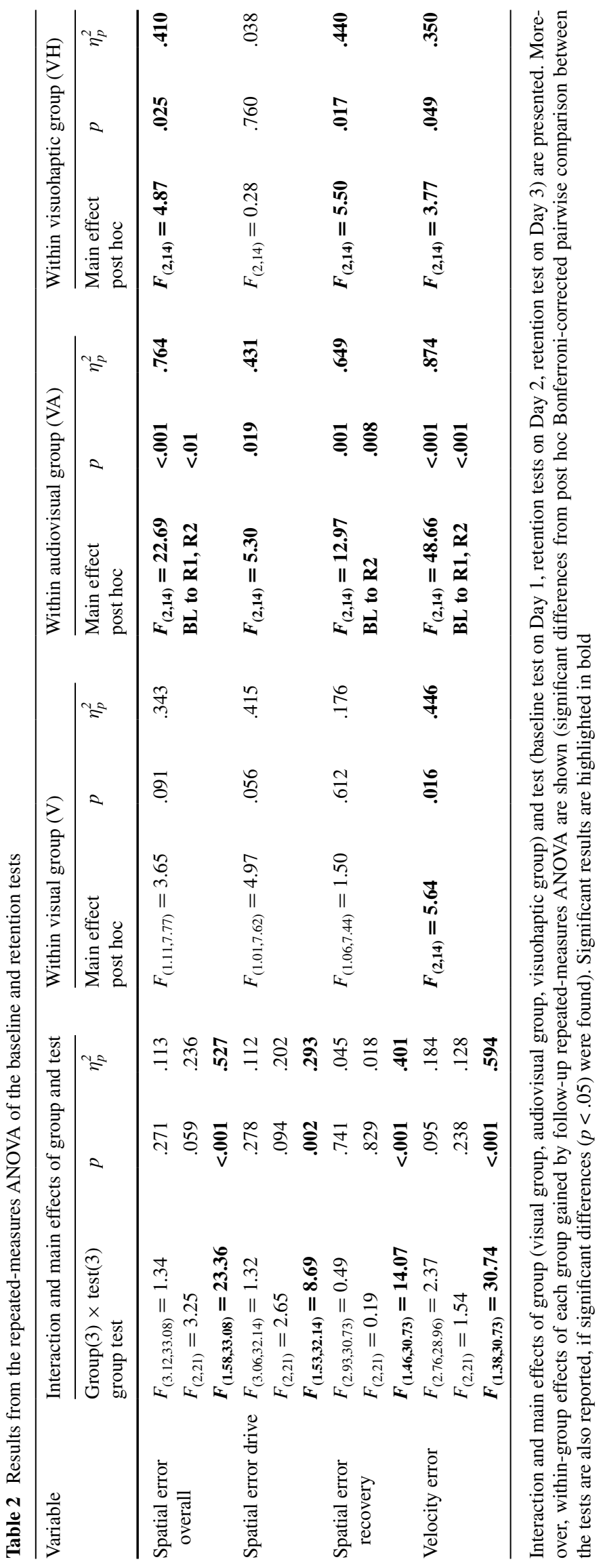




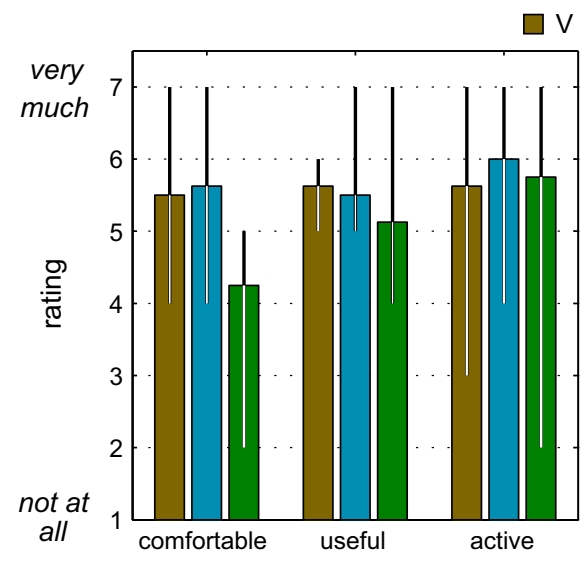

Fig. 6 Questionnaire answers. Left subjects' evaluation of the feedback designs (scale 1:7, 7 is best). Vertical black lines connect lowest and highest answer of a subject in a group. Right what subjects

Effects in the training sessions

From time to time, subjects of $\mathrm{V}$ and $\mathrm{VH}$ were observed to ignore the moving target oar for some cycles, which resulted in significantly higher velocity errors compared to the non-feedback trails. It seems that the focus of $\mathrm{V}$ and VH lied dominantly on staying on the path, i.e., on minimizing spatial errors, because the trace (and haptic reactive feedback for $\mathrm{VH}$ ) was clearer to perceive than the moving target oar. Concerning spatial aspects of the movement in the training sessions, all groups performed better with the feedback than without (Fig. 4; Table 1), supporting the guidance hypothesis (Salmoni 1984; Schmidt 1991; Schmidt et al. 1989). This is not surprising, even though the visual feedback was blurred and only provided beyond a deviation threshold. The feedback was clearly perceivable to perform the complex movement more precisely than in non-feedback trials.

Compared to V and VH, VA performed significantly better during feedback training concerning movement velocity (Fig. 4; Table 1), confirming an excellent "action-listing" mechanism (Lahav et al. 2007) fostered by movement sonification (Schaffert et al. 2011). As reported by others (Schaffert et al. 2011; Secoli et al. 2011), the auditory feedback guided the focus on a specific aspect, in this case toward the movement velocity as intended. VA showed no significant differences between feedback training and nonfeedback trials in the velocity error in contrast to $\mathrm{V}$ and $\mathrm{VH}$ (Fig. 4; Table 1), which indicated a transfer of the trained velocity to non-feedback conditions. Thus, movement sonification seemed not to evoke a dependency on the feedback, which contradicts the guidance hypothesis. Furthermore, the additional auditory feedback did not overload the subjects since the feedback was used to improve performance. Therefore, not only experts perform better with sonification

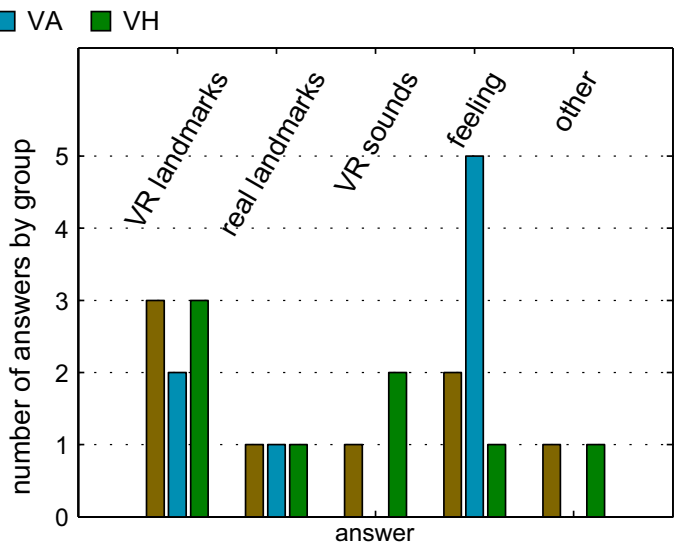

remembered primary for performing retention on Day 2 . The values indicate the number of subjects in a group that choose this answer. No subject answered "demonstration" or "real sounds"

of their own movement (Chollet et al. 1992; Schaffert et al. 2011), but also naïve subjects, at least if a reference movement is also sonified and played concurrently to the own, sonified movement, and if combined with visual feedback.

Not surprisingly, VH showed the highest velocity error during training with feedback as the reactive feedback hindered fluent movement execution when subjects deviated from the trajectory. The perceived reactive force may also have caused the worst rating in terms of comfort (Fig. 6). Interestingly, three subjects of the VH group had extremely high errors in feedback training regarding the velocity error even on Day 2, which was not observed in any other group (Fig. 4, right). Thus, the reactive feedback seems to be too challenging for some subjects and overload them, which was also observed in studies on error amplification (Cesqui et al. 2008; Milot et al. 2010). The augmented feedback (in our case the magnitude of the reactive force) might thus have been more effective if it is adapted to the skill level of the subject (Huegel and O'Malley 2010; Krebs et al. 2003; Marchal-Crespo and Reinkensmeyer 2008b; Sigrist et al. 2013a).

Learning from baseline to the retention tests

As hypothesized, all feedback designs were effective in terms of learning the complex movement. The learning curves from baseline to the retention tests of all groups showed a significant decrease (VA, VH) or a tendency (V) to decrease in overall spatial error and significant decrease in velocity error (Fig. 4; Table 2). It seemed that, as hypothesized, the visual feedback could intuitively instruct the novel, complex movement structure, which was indicated by an early error reduction from baseline to the non-feedback trials on Day 1. Thus, concurrent and frequent feedback does not necessarily hamper learning as postulated by 
the guidance hypothesis, if the feedback is well designed, i.e., the feedback should not overload the learner and allow the learner to process intrinsic feedback when the movement is performed correctly.

Indeed, the visual feedback allowed the subjects to remember the correct movement by integrating all kinds of information that were also available in non-feedback conditions. This was possible because target oar and trace disappeared when the movement was correct, similar to bandwidth feedback (Ribeiro et al. 2011; Timmermans et al. 2009). It seems that the visual feedback did not block parallel processing of visual and kinesthetic information (Wei and Körding 2009), calibration of proprioception (Buchanan and Wang 2012; Robin et al. 2005), proprioception of correct movements (Chiviacowsky and Wulf 2007; Winstein 1991), and the linkage of kinesthetic information with real and virtual landmarks (Sigrist et al. 2013a) (Fig. 6, right). Moreover, motivated by results of an earlier study (Sigrist et al. 2013b), the visual feedback was designed to avoid the correction of task-irrelevant errors (transparent target, deviation-dependent trace) that originate from noise in the sensory-motor system (Schmidt 1991). Thus, the visual feedback seemed to force learning of task-relevant aspects (trace width, trace color from red to green for increasing deviations). However, it remains unknown which aspects of the visual feedback design were indeed critical for successful learning and whether other thresholds and parameter settings can further enhance its effectiveness. Moreover, not only the design, but also the higher movement velocity compared to a prior study of the same task (Sigrist et al. 2013b) might have limited taskirrelevant, local corrections (Schmidt and Wulf 1997) and thus allowed for successful learning.

It seems that, as hypothesized, movement sonification in addition to visual feedback facilitates the perception of the movement velocity more than visual feedback alone, while avoiding cognitive overload. Even though it cannot be claimed that the setting of the mapping function parameters of the sonification was optimal for every subject at the current skill level, the benefits of movement sonification in addition to visual feedback were substantial. VA decreased the velocity error already in the first non-feedback trials on Day 1 to a level that was never reached by $\mathrm{VH}$ and only hardly by V later in the study, i.e., on Day 2 (see Fig. 4). Moreover, VA showed significant learning from baseline to both retention tests (Fig. 5; Table 2), the smallest velocity errors in the retention tests compared to $\mathrm{V}$ and $\mathrm{VH}$ (see Fig. 5), and a significantly higher baseline-corrected learning rate in velocity error than VH. Thus, movement sonification facilitated the movement in the retention tests, probably because the sound was kept in mind to perform the movement without feedback (Ronsse et al. 2011; Sigrist et al. 2013a). The applied movement sonification did not mask the real (or rendered) sound from the environment (Schaffert et al. 2011), e.g., water sound, which enabled a linkage of "auditory landmarks" to the motor task (Sigrist et al. 2013a). Perception of sound and action is strongly linked (Chen et al. 2008; Keysers and Gazzola 2010; Zatorre et al. 2007), whereby a sound can imply movements (Schaffert et al. 2011). The metrically organized sound can be anticipated and allows motor prediction (Zatorre et al. 2007). The subjects may have used the sonification as an auditory model, which helped to develop an internal representation of the target coordination pattern (Kennedy et al. 2013). Thus, the movement sonification became part of their internal movement representation and part of their feeling, on which five out of eight subjects of VA relied in the retention tests (Fig. 6).

Recent studies have applied haptic guidance to effectively teach dynamic or timing aspects of a movement (Lüttgen and Heuer 2012a, b, 2013, Marchal-Crespo et al. 2010, 2013; Milot et al. 2010). In this study, haptic feedback was used differently, i.e., the bidirectional properties of the haptic feedback were applied to prevent passivity of the subject. A common path controller would have allowed the subject to slide passively along the path walls. In contrast, the reactive feedback applied in this study forced the subjects to actively seek the correct path and to remember it in order to avoid the reactive forces. Thus, the haptic feedback aimed to enhance the awareness of the movement error while preventing the subjects to deviate too much from the reference path. In contrast to $\mathrm{V}$, and similarly to VA, VH significantly reduced overall spatial error and the spatial error during recovery. However, in general, the addition of the haptic reactive feedback neither showed higher learning rates nor smaller errors in the retention tests compared to the other groups (Fig. 5). The subjects that were highly challenged by the haptic feedback might have focused more on coping with the current reactive forces than on remembering how to avoid them in a next trial, i.e., they were overloaded. Thus, reactive feedback might be most effective for subjects who appeared to be initially more skilled (Cesqui et al. 2008; Milot et al. 2010) and might not have involved all subjects as intended. Indeed, VH did not report to be more (nor less) active compared to $\mathrm{V}$ or VA during feedback training (Fig. 6).

VH could significantly decrease the spatial error during recovery (and overall spatial error), but not during the drive phase (Fig. 5). In contrast to the drive phase, in the recovery phase, no rendered water resistance was interacting with the haptic feedback. Consequently, in the recovery phase, the reactive feedback seemed to be much better perceivable and, assumingly, also more involving. In the drive phase, the water resistance masked the reactive forces. Therefore, a linkage of landmarks to the motor task (Sigrist et al. 2013a) was facilitated only in the recovery phase. As 
concluded by others (Marchal-Crespo and Reinkensmeyer 2008a), it remains challenging to design effective and unambiguous haptic augmented feedback on top of rendered task forces in a virtual scenario.

The addition of the haptic feedback seemed not to be practical for all phases of the rowing cycle and not for all subjects, at least in the form it was applied in this study. The expected advantage of the haptic feedback, i.e., the fact that it could not be ignored might have been a disadvantage, at least for over-challenged subjects who were overwhelmed. The haptic feedback always disturbed the movement when performing poorly. This was not the case for the subjects of VA who were allowed to switch the focus from training with visual feedback to auditory feedback from time to time without being disturbed by the other modality. While they focused on the sonification, it can be assumed that the temporal aspects were dominantly perceived, i.e., the change in the frequency representing the movement velocity. Thus, the sonification provided redundant information to the visual feedback regarding movement velocity, however, in a more appropriate modality (Huang et al. 2005; Welch and Warren 1980), as audition is made to detect temporal information (Kapur et al. 2005; Kramer 1994). Intersensory facilitation (Carson and Kelso 2004) and multimodal integration (Alais and Burr 2004; Ernst and Banks 2002; van Beers et al. 1999) may have contributed to successful multimodal learning with audiovisual feedback (Seitz and Dinse 2007; Shams and Seitz 2008). The sonification also represented an absolute spatial variable, i.e., the horizontal oar angle. Thus, also spatial information was represented redundantly to the visual feedback. Indeed, learning of the spatial error during recovery was significant in VA (Table 2). Thus, the addition of movement sonification to the visual feedback allowed effective learning of spatial and temporal aspects in parallel.

\section{Conclusion and outlook}

The concurrent unimodal visual as well as the multimodal audiovisual and visuohaptic feedback designs in this study fostered learning of a complex, real-world task. In general, the effectiveness of augmented feedback might not exclusively depend on the feedback frequency. More likely, motor learning benefits from feedback that guides the focus of the learner on the source of information that is needed to recall the movement in conditions without feedback. It is likely that it is not the feedback modality per se that determines learning success, but the design of the feedback. In future studies, the contribution of specific parameters and features of the feedback designs to learning should be determined. Concerning the visual feedback, it remains unclear if the addition of a trace providing terminal feedback, blurring of the target, or the display of only the actual oar during correct movements allow transfer of skills to the non-feedback conditions. Furthermore, the mechanisms of the sonification that facilitate the recall of the movement after training should be identified. The potential of haptic feedback to involve a learner can be further exploited, also in combination with sonification.

The findings of this study confirm that conclusions concerning concurrent feedback and on the guidance hypothesis drawn from studies on a simple task do not necessarily transfer to complex tasks. Although laboratory tasks are low-dimensional, artificial, and therefore straightforward to investigate and very valuable for basic motor learning research, they do not represent movements in real life, which, by way of contrast, are commonly multidimensional, complex and performed in a real, contextual environment. Since the effort to conduct studies on the effectiveness of concurrent feedback for complex, real-life tasks learning is huge, investigation has been rare or even nonexistent regarding multimodal feedback. However, further studies on complex, real-life tasks could contribute to the optimization of augmented feedbacks in sports and rehabilitations. It should be tested whether conclusions drawn from the results of this study on learning trunk-arm rowing can be transferred to learning of other tasks in sports and rehabilitation.

Acknowledgments We want to thank Prof. Nicole Wenderoth for her enriching comments. We thank Alessandro Rotta, Pascal Wespe, and Marco Bader for their indispensable technical contributions to the design and setup of the simulator. Moreover, thanks go to Michael Herold-Nadig for his support on technical and safety issues and Mark van Raai for designing the visual feedback and rowing scenario. We want to thank Stefan Seiterle, Luca Lörtscher, Florian Kübler, and Nicolas Gerig for their help in conducting the measurements, and the subjects for participating. We thank Andrew Pennycott for proofreading the manuscript. This work was supported by ETH Zurich, the SNF-Grant "Impact of Different Feedback Modalities on Complex Skill Learning," CR22I2 135101/1, and the SNF-Grant "Acceleration of complex motor learning by skill level-dependent feedback design and automatic selection”, CR23I2_152817.

\section{References}

Alais D, Burr D (2004) The ventriloquist effect results from near-optimal bimodal integration. Curr Biol 14(3):257-262

Blandin Y, Toussaint L, Shea CH (2008) Specificity of practice: interaction between concurrent sensory information and terminal feedback. J Exp Psychol Learn Mem Cogn 34(4):994-1000

Braun DA, Mehring C, Wolpert DM (2010) Structure learning in action. Behav Brain Res 206(2):157-165

Brown R, Palmer C (2012) Auditory-motor learning influences auditory memory for music. Mem Cognit 40(4):567-578

Buchanan J, Wang C (2012) Overcoming the guidance effect in motor skill learning: feedback all the time can be beneficial. Exp Brain Res 219(2):305-320

Burke JL, Prewett MS, Gray AA, Yang L, Stilson FRB, Coovert MD, Elliot LR, Redden E (2006) Comparing the effects of 
visual-auditory and visual-tactile feedback on user performance: a meta-analysis. In: Proceedings of the 8th international conference on multimodal interfaces, New York, NY, USA, pp 108-117

Carson RG, Kelso JAS (2004) Governing coordination: behavioural principles and neural correlates. Exp Brain Res 154(3):267-274

Cesqui B, Aliboni S, Mazzoleni S, Carrozza M, Posteraro F, Micera $S$ (2008) On the use of divergent force fields in robot-mediated neurorehabilitation. In: 2nd IEEE RAS EMBS international conference on biomedical robotics and biomechatronics 2008 BioRob 2008, pp 854-861

Chen X, Agrawal S (2013) Assisting versus repelling force-feedback for learning of a line following task in a wheelchair. IEEE Trans Neural Syst Rehabil Eng 21(6):959-968

Chen JL, Penhune VB, Zatorre RJ (2008) Listening to musical rhythms recruits motor regions of the brain. Cereb Cortex 18(12):2844-2854

Chiviacowsky S, Wulf G (2007) Feedback after good trials enhances learning. Res Q Exerc Sport 78:40-47

Chollet D, Madani M, Micallef JP (1992) Effects of two types of biomechanical bio-feedback on crawl performance. In: MacLaren D, Reilly T, Lees A (eds) Biomechanics and medicine in swimming. E \& FN Spon, London, pp 57-62

Effenberg AO (2005) Movement sonification: effects on perception and action. IEEE Multimedia 12(2):53-59

Emken J, Reinkensmeyer DJ (2005) Robot-enhanced motor learning: accelerating internal model formation during locomotion by transient dynamic amplification. IEEE Trans Neural Syst Rehabil Eng 13(1):33-39

Ernst MO, Banks MS (2002) Humans integrate visual and haptic information in a statistically optimal fashion. Nature 415(6870):429-433

Freides D (1974) Human information processing and sensory modality: cross-modal functions information complexity memory and deficit. Psychol Bull 81(5):284-310

Giese MA, Poggio T (2000) Morphable models for the analysis and synthesis of complex motion patterns. Int $\mathrm{J}$ Comput Vis 38(1):59-73

Guadagnoli M, Kohl R (2001) Knowledge of results for motor learning: relationship between error estimation and knowledge of results frequency. J Mot Behav 33(2):217-224

Guadagnoli MA, Lee TD (2004) Challenge point: a framework for conceptualizing the effects of various practice conditions in motor learning. J Mot Behav 36(2):212-224

Hale K, Stanney K (2004) Deriving haptic design guidelines from human physiological psychophysical and neurological foundations. IEEE Comput Graph Appl 24(2):33-39

Holden MK (2005) Virtual environments for motor rehabilitation: review. Cyberpsychol Behav 8(3):187-211

Huang F, Patton J (2013) Augmented dynamics and motor exploration as training for stroke. IEEE Trans Biomed Eng 60(3):838-844

Huang H, Ingalls T, Olson L, Ganley K, Rikakis T, He J (2005) Interactive multimodal biofeedback for task-oriented neural rehabilitation. In: 27 th annual international conference of the engineering in medicine and biology society 2005 IEEE-EMBS 2005, Shanghai, pp 2547-2550

Hubbard T (2013) Auditory imagery contains more than audition. In: Lacey S, Lawson R (eds) Multisensory imagery. Springer, New York, pp 221-247

Huegel J, O'Malley MK (2010) Progressive haptic and visual guidance for training in a virtual dynamic task. In: haptics symposium 2010 IEEE, pp 343-350

Israel J, Campbell D, Kahn J, Hornby T (2006) Metabolic costs and muscle activity patterns during robotic-and therapist-assisted treadmill walking in individuals with incomplete spinal cord injury. Phys Ther 86(11):1466-1478
Kapur A, Tzanetakis G, Virji-Babul N, Wang G, Cook PR (2005) A framework for sonification of vicon motion capture data. In: Proceedings of the 8 th conference on digital audio effects, Madrid, Spain

Kennedy D, Boyle J, Shea C (2013) The role of auditory and visual models in the production of bimanual tapping patterns. Exp Brain Res 224(4):507-518

Keysers C, Gazzola V (2010) Social neuroscience: mirror neurons recorded in humans. Curr Biol 20(8):R353-R354

Kim RS, Seitz AR, Shams L (2008) Benefits of stimulus congruency for multisensory facilitation of visual learning. PLoS One 3(1):e1532

Kovacs AJ, Shea CH (2011) The learning of $90^{\circ}$ continuous relative phase with and without lissajous feedback: external and internally generated bimanual coordination. Acta Psychol 136(3):311-320

Krakauer J, Mazzoni P (2011) Human sensorimotor learning: adaptation skill and beyond. Curr Opin Neurobiol 21(4):636-644

Kramer G (1994) Auditory display: sonification audification and auditory interfaces. Addison-Wesley, Reading MA

Krebs HI, Palazzolo JJ, Dipietro L, Ferraro M, Krol J, Rannekleiv K, Volpe BT, Hogan N (2003) Rehabilitation robotics: performance-based progressive robot-assisted therapy. Auton Robots 15(1):7-20

Lahav A, Saltzman E, Schlaug G (2007) Action representation of sound: audiomotor recognition network while listening to newly acquired actions. J Neurosci 27(2):308-314

Liebermann DG, Katz L, Hughes MD, Bartlett RM, McClements J, Franks IM (2002) Advances in the application of information technology to sport performance. J Sports Sci 20(10):755-769

Liu D, Todorov E (2007) Evidence for the flexible sensorimotor strategies predicted by optimal feedback control. J Neurosci 27(35):9354-9368

Liu J, Wrisberg CA (1997) The effect of knowledge of results delay and the subjective estimation of movement form on the acquisition and retention of a motor skill. Res Q Exerc Sport 68(2):145-151

Lüttgen J, Heuer H (2012a) The influence of haptic guidance on the production of spatio-temporal patterns. Hum Mov Sci 31(3):519-528

Lüttgen J, Heuer H (2012b) Robotic guidance benefits the learning of dynamic but not of spatial movement characteristics. Exp Brain Res 222(1-2):1-9

Lüttgen J, Heuer H (2013) The influence of robotic guidance on different types of motor timing. J Mot Behav 45(3):249-258

Marchal-Crespo L, Reinkensmeyer DJ (2008a) Effect of robotic guidance on motor learning of a timing task. In: 2nd IEEE RAS EMBS international conference on biomedical robotics and biomechatronics 2008 BioRob 2008, pp 199-204

Marchal-Crespo L, Reinkensmeyer DJ (2008b) Haptic guidance can enhance motor learning of a steering task. J Mot Behav 40(6):545-557

Marchal-Crespo L, Reinkensmeyer DJ (2009) Review of control strategies for robotic movement training after neurologic injury. J Neuroeng Rehabil 6(1):20

Marchal-Crespo L, Furumasu J, Reinkensmeyer DJ (2010) A robotic wheelchair trainer: design overview and a feasibility study. J Neuroeng Rehabil 7(1):40-51

Marchal-Crespo L, Raai M, Rauter G, Wolf P, Riener R (2013) The effect of haptic guidance and visual feedback on learning a complex tennis task. Exp Brain Res 231(3):277-291

Marschall F, Bund A, Wiemeyer J (2007) Does frequent feedback really degrade learning? A meta analysis. E-Journal Bewegung und Training 1:75-86

Milot MH, Marchal-Crespo L, Green CS, Cramer SC, Reinkensmeyer DJ (2010) Comparison of error-amplification and haptic-guidance 
training techniques for learning of a timing-based motor task by healthy individuals. Exp Brain Res 201(2):119-131

Minogue J, Jones MG (2006) Haptics in Education: exploring an untapped sensory modality. Rev Educ Res 76(3):3-17

Patton JL, Stoykov M, Kovic M, Mussa-Ivaldi F (2006) Evaluation of robotic training forces that either enhance or reduce error in chronic hemiparetic stroke survivors. Exp Brain Res 168(3):368-383

Patton JL, Wei YJ, Bajaj P, Scheidt RA (2013) Visuomotor learning enhanced by augmenting instantaneous trajectory error feedback during reaching. PLoS One 8(1):e46466

Proteau L (1992) On the specificity of learning and the role of visual information for movement control. In: Proteau L, Elliott D (eds) Vision and motor control, vol 85. North-Holland, Amsterdam, pp 67-103

Rauter G, von Zitzewitz J, Duschau-Wicke A, Vallery H, Riener R (2010) A tendon based parallel robot applied to motor learning in sports. In: 3rd IEEE RAS and EMBS international conference on biomedical robotics and biomechatronics (BioRob) 2010, Tokyo, Japan, pp 82-87

Rauter G, Sigrist R, Marchal-Crespo L, Vallery H, Riener R, Wolf P (2011) Assistance or challenge? Filling a gap in user-cooperative control. In: IEEE/RSJ international conference on intelligent robots and systems (IROS), pp 3068-3073

Rauter G, Sigrist R, Koch C, Crivelli F, van Raai M, Riener R, Wolf P (2013) Transfer of complex skill learning from virtual to real rowing. PLoS One 8(12):1-18

Reinkensmeyer DJ, Akoner O, Ferris D, Gordon K (2009) Slacking by the human motor system: computational models and implications for robotic orthoses. In: Engineering in medicine and biology society 2009 EMBC 2009 annual international conference of the IEEE, pp 2129-2132

Ribeiro DC, Sole G, Abbott JH, Milosavljevic S (2011) Extrinsic feedback and management of low back pain: a critical review of the literature. Man Ther 16(3):231-239

Robin C, Toussaint L, Blandin Y, Proteau L (2005) Specificity of learning in a video-aiming task: modifying the salience of dynamic visual cues. J Mot Behav 37(5):367-376

Ronsse R, Puttemans V, Coxon JP, Goble DJ, Wagemans J, Wenderoth N, Swinnen SP (2011) Motor learning with augmented feedback: modality-dependent behavioral and neural consequences. Cereb Cortex 21(6): 1283-1294

Salmoni S (1984) Knowledge of results and motor learning A review and critical reappraisal. Psychol Bull 95(3):355-386

Schaffert N, Mattes K, Effenberg AO (2011) An investigation of online acoustic information for elite rowers in on-water training conditions. J Hum Sport Exerc 6(2):392-405

Schmidt RA (1991) Frequent augmented feedback can degrade learning: evidence and interpretations. Tutor Motor Neurosci 62:59-75

Schmidt RA, Wrisberg C (2008) Motor learning and performance: a situation-based learning approach. Human Kinetics, Champaign, IL

Schmidt RA, Wulf G (1997) Continuous concurrent feedback degrades skill learning: implications for training and simulation. Hum Factors 39(4):509-525

Schmidt RA, Young DE, Swinnen S, Shapiro DC (1989) Summary knowledge of results for skill acquisition: support for the guidance hypothesis. J Exp Psychol Learn Mem Cogn 15(2):352-359

Schmitz G, Mohammadi B, Hammer A, Heldmann M, Samii A, Munte T, Effenberg A (2013) Observation of sonified movements engages a basal ganglia frontocortical network. BMC Neurosci 14(1):1-11

Secoli R, Milot M, Rosati G, Reinkensmeyer DJ (2011) Effect of visual distraction and auditory feedback on patient effort during robot-assisted movement training after stroke. J Neuroeng Rehabil $8(1): 1-10$
Seitz AR, Dinse HR (2007) A common framework for perceptual learning. Curr Opin Neurobiol 17(2):148-153

Seitz AR, Kim R, Shams R (2006) Sound facilitates visual learning. Curr Biol 16(14):1422-1427

Shams L, Seitz AR (2008) Benefits of multisensory learning. Trends Cogn Sci 12(11):411-417

Sigrist R, Schellenberg J, Rauter G, Broggi S, Riener R, Wolf P (2011) Visual and auditory augmented concurrent feedback in a complex motor task. Presence Teleop Virt 20(1):15-32

Sigrist R, Rauter G, Riener R, Wolf P (2013a) Augmented visual auditory haptic and multimodal feedback in motor learning: a review. Psychon Bull Rev 20:21-53

Sigrist R, Rauter G, Riener R, Wolf P (2013b) Terminal feedback outperforms concurrent visual auditory and haptic feedback in learning a complex rowing-type task. J Mot Behav 45(6):455-472

Snodgrass SJ, Rivett DA, Robertson VJ, Stojanovski E (2010) Realtime feedback improves accuracy of manually applied forces during cervical spine mobilization. Man Ther 15:19-25

Swinnen SP, Schmidt RA, Nicholson DE, Shapiro DC (1990) Information feedback for skill acquisition: instantaneous knowledge of results degrades learning. J Exp Psychol Learn Mem Cogn 16(4):706-716

Swinnen SP, Lee TD, Verschueren S, Serrien DJ, Bogaerds H (1997) Interlimb coordination: learning and transfer under different feedback conditions. Hum Mov Sci 16(6):749-785

Thoroughman K, Shadmehr R (2000) Learning of action through adaptive combination of motor primitives. Nature 407(6805):742

Timmermans AAA, Seelen HAM, Willmann RD, Kingma H (2009) Technology-assisted training of arm-hand skills in stroke: concepts on reacquisition of motor control and therapist guidelines for rehabilitation technology design. J Neuroeng Rehabil 6:1

Todorov E (2004) Optimality principles in sensorimotor control. Nat Neurosci 7:907-915

Todorov E, Jordan MI (2002) Optimal feedback control as a theory of motor coordination. Nat Neurosci 5:1226-1235

Todorov E, Shadmehr R, Bizzi E (1997) Augmented feedback presented in a virtual environment accelerates learning of a difficult motor task. J Mot Behav 29(2):147-158

van Beers RJ (2009) Motor learning is optimally tuned to the properties of motor noise. Neuron 63(3):406-417

van Beers RJ, Sittig AC, Gon JJ (1999) Integration of proprioceptive and visual position-information: an experimentally supported model. J Neurophysiol 81(3):1355

van der Linden DW, Cauraugh JH, Greene TA (1993) The effect of frequency of kinetic feedback on learning an isometric force production task in nondisabled subjects. Phys Ther 73(2):79-87

Vlachos M, Hadjieleftheriou M, Gunopulos D, Keogh E (2003) Indexing multi-dimensional time-series with support for multiple distance measures. In: proceedings of the ninth acm sigkdd international conference on knowledge discovery and data mining KDD’03, New York, NY, USA, pp 216-225

von Zitzewitz J, Wolf P, Novakovic V, Wellner M, Rauter G, Brunschweiler A, Riener R (2008) Real-time rowing simulator with multimodal feedback. Sports Technol 1(6):257-266

Wei K, Körding K (2009) Relevance of error: what drives motor adaptation? J Neurophysiol 101(2):655-664

Welch RB, Warren DH (1980) Immediate perceptual response to intersensory discrepancy. Psychol Bull 88(3):638-667

Wickens CD (2002) Multiple resources and performance prediction. Theor Issues Ergon Sci 3(2):159-177

Winstein CJ (1991) Knowledge of results and motor learning-implications for physical therapy. Phys Ther 71(2):140-149

Winstein CJ, Pohl PS, Cardinale C, Green A, Scholtz L, Waters CS (1996) Learning a partial-weight-bearing skill: effectiveness of two forms of feedback. Phys Ther 76(9):985-993 
Wishart LR, Lee TD, Cunningham SJ, Murdoch JE (2002) Agerelated differences and the role of augmented visual feedback in learning a bimanual coordination pattern. Acta Psychol (Amst) 110(2-3):247-263

Wolpert D, Flanagan J (2010) Motor learning. Curr Biol 20(11): R467-R472

Wolpert DM, Diedrichsen J, Flanagan JR (2011) Principles of sensorimotor learning. Nat Rev Neurosci 12:739-749

Wulf G (2007) Self-controlled practice enhances motor learning: implications for physiotherapy. Physiotherapy 93(2):96-101
Wulf G, Shea CH (2002) Principles derived from the study of simple skills do not generalize to complex skill learning. Psychon Bull Rev 9(2):185-211

Wulf G, Shea CH, Matschiner S (1998) Frequent feedback enhances complex motor skill learning. J Mot Behav 30(2):180-192

Wulf G, Hörger M, Shea CH (1999) Benefits of blocked over serial feedback on complex motor skill learning. J Mot Behav 31(1):95-103

Zatorre RJ, Chen JL, Penhune VB (2007) When the brain plays music: auditory-motor interactions in music perception and production. Nat Rev Neurosci 8(7):547-558 